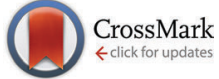

Cite this: Phys. Chem. Chem. Phys., 2014, 16, 22255

Received 25th May 2014, Accepted 26th August 2014

DOI: 10.1039/c4cp02295e

www.rsc.org/pccp

\title{
Chemistry-dependent X-ray-induced surface charging
}

\author{
Bekir Salgın, $\dagger^{\star{ }^{a}}$ Diego Pontoni, ${ }^{b}$ Dirk Vogel, ${ }^{a}$ Heiko Schröder, † $^{\mathrm{c}}$ Patrick Keil, $\S^{\mathrm{a}}$ \\ Martin Stratmann, ${ }^{a}$ Harald Reichert ${ }^{\mathrm{bc}}$ and Michael Rohwerder ${ }^{\mathrm{a}}$
}

\begin{abstract}
Materials science in general, and surface/interface science in particular, have greatly benefited from the development of high energy synchrotron radiation facilities. Irradiation with intense ionizing beams can however influence relevant sample properties. Permanent radiation damage and irradiation-induced sample modifications have been investigated in detail during the last decades. Conversely, reversible sample alterations taking place only during irradiation are still lacking comprehensive in situ characterization. Irradiation-induced surface charging phenomena are particularly relevant for a wide range of interface science investigations, in particular those involving surfaces of solid substrates in contact with gaseous or liquid phases. Here, we demonstrate partially reversible radiation-induced surface charging phenomena, which extend far beyond the spatial dimensions of the X-ray beam mainly as a consequence of the interaction between the surface and ionized ambient molecules. The charging magnitude and sign are found to be surface chemistry specific and dependent on the substrates' bulk conductivity and grounding conditions. These results are obtained by combining a scanning Kelvin probe with a synchrotron surface diffractometer to allow simultaneous in situ work function measurements during precisely controlled hard X-ray micro-beam irradiation.
\end{abstract}

\section{Introduction}

Progress in surface science is increasingly stemming from the exploitation of synchrotron radiation ${ }^{1}$ due to its superior properties (e.g., high brilliance, energy tunability, partial coherence) and prospected advances. ${ }^{2}$ The integration of well-established surface and bulk characterization techniques with state-of-theart synchrotron beamlines enables high resolution characterization of a wide range of materials. ${ }^{3-5}$ The availability of ever more intense hard X-ray synchrotron beams requires, however, detailed evaluation of the effects of X-ray irradiation onto the measured sample properties. For instance, first reports on the susceptibility of organic samples (e.g., proteins) to radiation damage date back to the earliest days of synchrotron experiments. ${ }^{6}$

\footnotetext{
${ }^{a}$ Max-Planck-Institut für Eisenforschung GmbH, Max-Planck-Strasse 1, 40237 Duesseldorf, Germany. E-mail: salgin@mpie.de

${ }^{b}$ European Synchrotron Radiation Facility, 6, rue Jules Horowitz, 38043 Grenoble, France

${ }^{c}$ Max-Planck-Institut für Metallforschung, Heisenbergstrasse 3, 70569 Stuttgart, Germany

$\dagger$ Present address: Stryker Trauma GmbH, Prof.-Küntscher-Strasse 1-5, 24232 Schönkirchen, Germany.

‡ Present address: Tesat-Spacecom GmbH \& Co. KG, Gerberstrasse 49, 71522 Backnang, Germany.

$\S$ Present address: BASF Coatings GmbH, Glasuritstrasse 1, 48165 Münster, Germany.
}

During the following decades, the increased availability of synchrotron user facilities allowed detailed studies of radiation damage $^{7-11}$ leading to refined strategies for its mitigation. 9,12,13 The most evident effects of radiation damage are permanent structural alterations ${ }^{10,14,15}$ that should be carefully monitored to avoid data misinterpretation. Reversible sample modifications that occur only during X-ray exposure are potentially even more deleterious for reliable data evaluation. In situ detection of such modifications becomes therefore of utmost importance for synchrotron-based surface and interface science investigations.

It is well known that a sample surface charges when it is directly exposed to an X-ray beam. ${ }^{8,16}$ In the hard X-ray regime, surface charging is the result of a complex interplay between photoelectric absorption, Auger emission and Compton scattering processes. The electric fields building up as a consequence of surface charging can directly affect the orientation and packing of adsorbed molecules and molecular aggregates. ${ }^{17-19}$ For instance, the structure of double layers in electrochemical systems is governed by beam induced interface charging, as e.g. observed for ionic liquids on sapphire. ${ }^{20} \mathrm{X}$-ray-induced surface charging can be completely or partially reversible, therefore ex situ surface analysis after synchrotron experiments is of limited interest and potentially misleading. We therefore implemented a scanning Kelvin probe (SKP) specifically designed for the in situ monitoring of surface and interface electrostatic properties during synchrotron investigations. ${ }^{21}$ 
The Kelvin probe is a non-destructive, non-contact technique ${ }^{22}$ for measuring the work function. Considering the sensitivity of the method for even the slightest net charging, ${ }^{23}$ it is a suitable tool for detecting work function variations induced by surface charging phenomena. Although, by definition, charging should not affect the work function of a sample, for insulating or semiconducting samples it causes the misalignment of the Fermi level, which manifests itself in the Kelvin probe measurement as an apparent decrease/increase of the work function for positive/negative net charging of the surfaces ${ }^{24}$ (see discussion below). The technique was already used for sensing surface photovoltage variations at the X-ray beam illumination footprint on $\mathrm{GaAs}^{25-28}$ and $\mathrm{GaP}(110) / \mathrm{Ag}$ films. ${ }^{29}$ In a recent ex situ study, significant surface potential changes, after vacuum ultraviolet (VUV) exposure, were reported for various dielectric materials. ${ }^{30,31}$ However, detailed in situ studies featuring sufficient lateral resolution for resolving the zone affected by the X-ray beam are still missing. In addition, the role of different surface chemistries on beam induced charging effects was not to date investigated quantitatively and systematically.

In this study, we demonstrate that X-ray induced surface charging extends spatially well beyond the beam footprint region mainly as a consequence of the interaction of ionized air molecules with the surface. Variation of the surface chemistry, substrate bulk conductivity, and grounding methods affect the charging phenomena both qualitatively and quantitatively. Our approach enables detailed studies of all factors involved in irradiation induced surface charging phenomena, thus providing the means to control and monitor in situ their magnitude and spatial extent.

\section{Experimental}

The SKP apparatus, specifically designed for synchrotron applications, was integrated ${ }^{21}$ with the High Energy Micro-Diffraction setup $^{32,33}$ of the ID15A beamline of the European Synchrotron Radiation Facility (ESRF). The $72.5 \mathrm{keV}$ X-ray beam used to

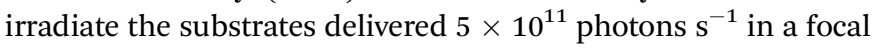
spot of $5 \times 20 \mu \mathrm{m}(V \times H)$. The sample surface was carefully aligned with the fixed, horizontal X-ray beam (incidence angle $\alpha_{i}=0 \pm 0.0001^{\circ}$ ). A high resolution sample translation stage allowed variation of the surface-to-beam vertical separation with sub- $\mu \mathrm{m}$ accuracy. The exposure protocol comprised a controlled sequence of vertical sample displacements allowing irradiation of the bulk substrate at various depths $d$ below the surface (Fig. 1A), namely: (a) X-ray beam switching on at $d=250 \mu \mathrm{m}$, (b) consecutive $50 \mu \mathrm{m}$ downwards sample movements until $d=0 \mu \mathrm{m}$, (c) direct surface irradiation during 3 to 8 minutes, (d) reversed, $50 \mu \mathrm{m}$ step, upwards sample scan, (e) X-ray beam switching off at $d=250 \mu \mathrm{m}$. The sample scans lasted typically 40 to 60 minutes. The $100 \mu \mathrm{m}$ diameter cylindrical tip of the Kelvin probe was continuously scanned back and forth above the surface perpendicularly to the beam ( $x$-direction in Fig. 1A). The vertical tip-to-surface gap was $50 \mu \mathrm{m}$ and the scan range was $1 \mathrm{~mm}$, centered along the beam direction. The SKP scans thus provided time-resolved one-dimensional work-function profiles before, during and after X-ray irradiation (Fig. 1B and D). Occasionally, negative $d$ values were investigated in order to compare the effects of the beam passing through air above the sample surface and through the bulk substrate below it. Due to the high X-ray energy used, the beam was only partially attenuated when traversing the bulk substrate. Samples consisted of square approximately $10 \times 10 \mathrm{~mm}^{2}$ substrates cut from $1 \mathrm{~mm}$ thick wafers of insulating, semiconducting and conducting materials, namely: sapphire, p-type and n-type silicon, gold-coated p-type silicon, and NiAl alloy. Silicon samples comprised a native surface silica $\left(\mathrm{SiO}_{2}\right)$ layer of thicknesses in the range of 6 to $15 \AA$.

Just before the synchrotron experiments, silicon and sapphire samples were subjected to solvent cleaning followed by treatment in acidic or alkaline piranha solutions. Treating amphoteric metal oxides in solutions having $\mathrm{pH}$ values different from their isoelectric point (IP) induces fixed charged surface functional groups. For silica (IP at $\mathrm{pH} \sim 2$ ) acidic piranha creates positive fixed surface charges, while alkaline piranha induces negative surface charges. This change of the surface chemistry is clearly demonstrated by X-Ray Photoelectron Spectroscopy (XPS) analysis of the surface oxygen presented in Fig. 2. The alkaline treated sample features a distinct low energy shoulder related to negatively charged functional groups $\left(\mathrm{SiO}^{-}\right) \cdot{ }^{34,35}$ The intensity of this feature increases at lower take-off angles, therefore confirming that it originates from the surface. On the other hand, the low energy XPS component is absent in acidically treated silicon, because in this case protonated groups $\left(\mathrm{SiOH}_{2}{ }^{+}\right)$carry the fixed positive surface charges. ${ }^{34,35}$ The experiments were conducted at ambient condition (air conditioned, i.e. RT and low relative humidity), which enables the compensation of the charging resulting from these treatment-induced functional groups by counter charges adsorbed from the surrounding atmosphere. ${ }^{36}$ Therefore in the initial stage, (i.e., before the beam exposure) both surfaces are net uncharged, although they exhibit slightly different work functions as revealed by ex situ Kelvin probe measurements $\left(-130 \pm 50 \mathrm{mV}_{\mathrm{SHE}}\right.$ and $+60 \pm 20 \mathrm{mV}_{\mathrm{SHE}}$, for the acidically and alkaline treated surfaces respectively). These differences are small compared to the variations caused by exposure to the X-ray beam. More detailed sample preparation procedures are reported in the Materials and methods section.

\subsection{Materials and methods}

The boron-doped p-type Si wafers (Siltronic AG, Munich, Germany) had a resistivity of $10-20 \Omega \mathrm{cm}$ and (100) surface orientation. Samples were pretreated in freshly prepared acidic $(10 \mathrm{~min}$ in $1: 1, \mathrm{H}_{2} \mathrm{SO}_{4}: \mathrm{H}_{2} \mathrm{O}_{2}(35 \mathrm{wt} \%$ in water)) or alkaline (30 and $5 \mathrm{~min}$ in two steps, $1: 1, \mathrm{NH}_{4} \mathrm{OH}(28-30 \%$, Merck KGaA, Darmstadt, Germany) $: \mathrm{H}_{2} \mathrm{O}_{2}$ ) piranha solutions after being cleaned by subsequent ultrasonications in ethanol, isopropanol, chloroform (15 min each). After both pretreatments, the samples were rinsed with a stream of ultrapure argon. The chosen piranha solution pretreatments are efficient for cleaning surfaces especially from organic contaminations. To serve as an electrical contact, the bottom side of the sample labelled as "p-type Si wafer with improved contact" was metallized with a $\sim 300 \mathrm{~nm}$ thick gold layer. The sample was pretreated in acidic piranha solution. 

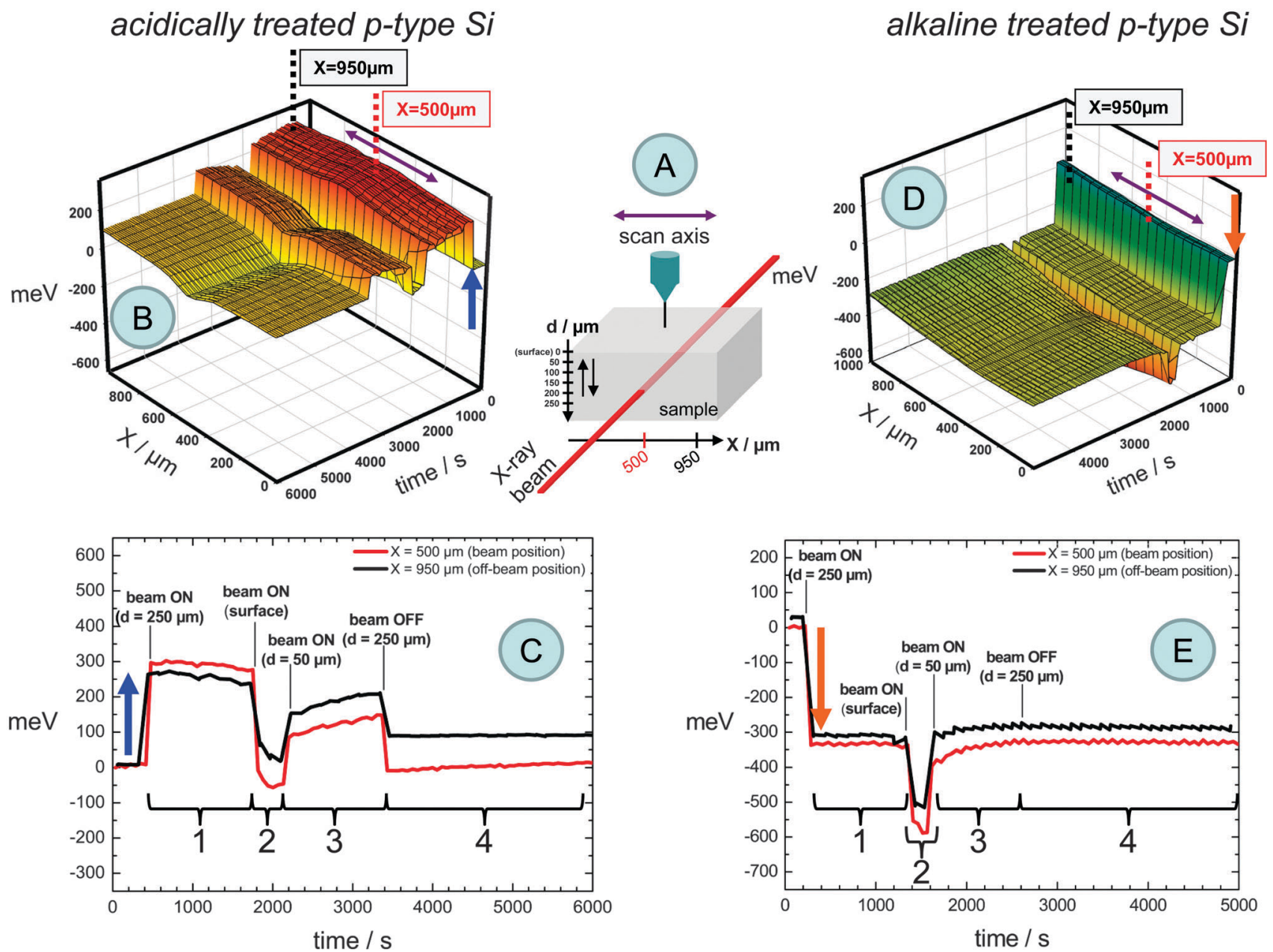

Fig. $1 \mathrm{X}$-ray-induced work function variations for $\mathrm{p}$-type silicon under varying irradiation conditions and surface pre-treatments. (A) Schematics of the irradiation protocol: at switching on, the fixed horizontal beam traverses the bulk sample $250 \mu \mathrm{m}$ below its surface; the sample is then lowered stepwise until the beam reaches the surface; after irradiating the surface for some minutes the sample is raised stepwise and the beam is switched off when it reaches again a depth of $250 \mu \mathrm{m}$. (B) Acidic pre-treatment, time-resolved work function profiles during the vertical sample movements. (C) Work function traces from (B) at beam (red line) and off-beam (black line) positions. The numbered time ranges indicate periods during which (1) the beam depth $d$ is decreased at $50 \mu \mathrm{m}$ steps, (2) the beam impinges directly on the surface, (3) $d$ is increased stepwise back to $250 \mu \mathrm{m}$, and (4) the beam is switched off. (D) Alkaline pre-treatment, time-resolved work function profiles during the vertical sample movements. (E) Work function traces from (D) at beam (red line) and off-beam (black line) positions.

The phosphorus-doped n-type Si wafer had a resistivity of 7-13 $\Omega \mathrm{cm}$ and (111) surface orientation. It underwent the same acidic piranha pretreatment described previously.

The polished NiAl single crystal alloy had (110) surface orientation. The sole pretreatment consisted in dipping consecutively for 10 minutes in isopropanol, acetone and chloroform. The sample was subsequently rinsed with ultrapure water and dried with a stream of ultrapure argon.

The sapphire single crystal was first dipped consecutively in isopropanol, acetone, chloroform for $15 \mathrm{~min}$ each. It was then pretreated for 3 min with acidic piranha, as described previously. Finally it was rinsed for $5 \mathrm{~min}$ with ultrapure water and dried with a stream of ultrapure argon.

All chemicals were supplied by Sigma-Aldrich Chemie GmbH (Munich, Germany), if not otherwise stated.

2.1.1 XPS. XPS measurements were performed with a Quantum 2000 (Physical Instruments, USA) using a monochromated
Al $\mathrm{K}_{\alpha} \mathrm{X}$-ray source with a spot diameter of $100 \mu \mathrm{m}$ and a pass energy of $11.75 \mathrm{eV}$ for the high resolution photoelectron spectra. The take-off angle of the detected photoelectrons was varied from $10^{\circ}$ to $80^{\circ}$ with respect to the surface. The calibration of the spectra was performed using the $\mathrm{C} 1 \mathrm{~s}$ peak of the $\mathrm{C}-\mathrm{C}$ photoelectron transition (binding energy, $\mathrm{BE}=285 \mathrm{eV}$ ) as internal reference.

\section{Results and discussion}

The effects of X-ray irradiation on p-type silicon are summarized in Fig. 1. The upper panels report the complete sequence of SKP scans during vertical sample movements. The lower panels present the corresponding time-resolved work function traces at the beam $(x=500 \mu \mathrm{m}$, red lines $)$ and off-beam $(x=950 \mu \mathrm{m}$, black lines) positions. In general, an increase of work function 


\section{A acidically treated $\mathrm{Si}$}

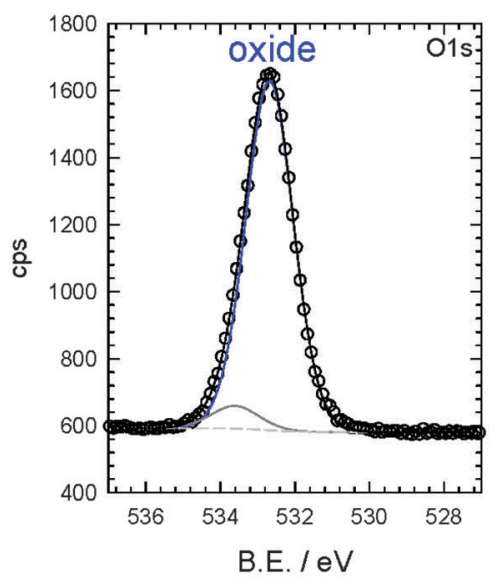

\section{B alkaline treated $\mathrm{Si}$}

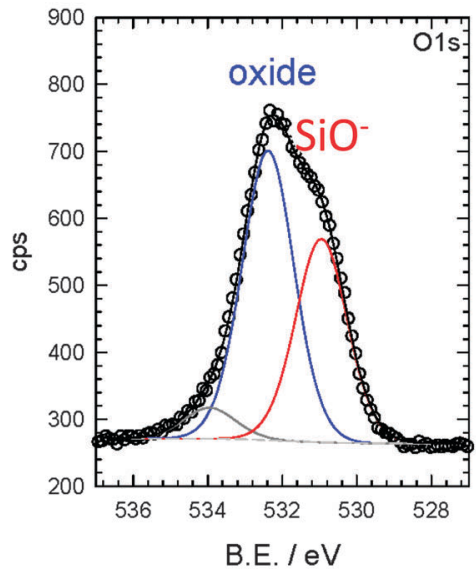

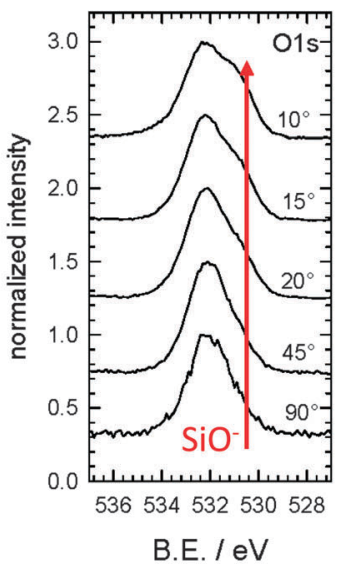

Fig. 2 High-resolution XPS spectra of the surface oxygen for (A) acidically treated and (B) alkaline treated p-type Si. The right-most panel reports the progressively increasing asymmetry of the XPS spectra with decreasing take-off angle thus confirming the surface nature of the negatively charged functional groups.

indicates that the surface becomes more negatively charged. When the beam is switched on, the work function of the acidically treated silicon increases instantaneously by about $250 \mathrm{meV}$ (blue arrows), while for the alkaline treated sample it decreases by about $350 \mathrm{meV}$ (orange arrows). Similar work function variations are measured also when the beam passes through air just above the surface $(d=-250 \mu \mathrm{m})$. Decreasing beam depth $d$ (regions "1" in Fig. 1C and E) has little or no effect on the work function. Direct surface irradiation at $d=0 \mu \mathrm{m}$ (regions " 2 " in Fig. 1C and E) causes a decrease of work function by $300-350 \mathrm{meV}$ in both acidically and alkaline treated samples. Stepwise return of the beam towards the bulk (regions "3") lifts the observed decrease resulting in partial work function recovery in the acidic case (Fig. 1C) and almost full recovery in the alkaline case (Fig. 1E). When the beam is finally switched off at $d=250 \mu \mathrm{m}$, the work function decreases rapidly for the acidically treated silicon, while it remains unchanged for the alkaline treated one. The work function is always slightly more negative at the beam position, with the exception of the first phase of the experiment on acidically treated Si (region " 1 " in Fig. 1C). Long after switching off the beam $(5000-6000 \mathrm{~s}$ in Fig. 1B and C) the initial $(t=0 \mathrm{~s})$ work function at the beam position $(x=500 \mu \mathrm{m})$ is almost recovered in acidically treated Si. Conversely, alkaline treated $\mathrm{Si}$ exhibits a permanent negative work function shift. Therefore beam-induced surface charging appears to be almost fully reversible for the acidic pre-treatment and largely irreversible for the alkaline one.

The above observations can be rationalized by considering the main types of charges involved in the experimental system: (a) the fixed, charged surface functional groups created by the chemical pre-treatment, (b) the abundant additional ionized molecules created in the surrounding atmosphere once the $\mathrm{X}$-ray beam is switched on, (c) the highly mobile majority charge carriers present in the bulk semiconductor - positive holes in p-type silicon - which act as mirror charges with respect to the surface-adsorbed ionized air molecules, and (d) the emitted photoelectrons inducing positive surface charging once the beam reaches the surface (Fig. 3).

When the X-ray beam is first switched on, ionized air molecules bearing a charge opposite to that of the fixed functional groups rapidly adsorb on the surface. This explains the different sign of the initial work function variations for acidic versus alkaline pre-treatments (blue and orange arrows in Fig. 1). A similar effect is observed in charged polymer systems, where charge carriers of sign opposite to that of the polymer exhibit high mobility, ${ }^{24,37}$ while charge carriers with the same sign experience the so-called Donnan exclusion..$^{38}$ As already mentioned above, although, by definition, charging should not affect the work function of a sample, for insulating or semiconducting samples it causes the misalignment of the Fermi level, which manifests itself in the Kelvin probe measurement as an apparent decrease/increase of the work function for positive/negative net charging of the surfaces, ${ }^{24}$ as observed here. The mirror charges present in the bulk silicon beneath the native silicon oxide layer compensate the surface charges absorbed from air (Fig. 3A) by delocalizing the net charge to the bottom of the sample (insulating oxide layer in contact with the base plate).

The abrupt work function decrease observed in both samples when $d=0 \mu \mathrm{m}$ indicates positive surface charging that originates from the emission of photoelectrons (Fig. 3B). Although the work function decrease is slightly more pronounced at the beam location, it actually extends over the entire probed area $(1 \mathrm{~mm})$, well beyond the beam footprint lateral width of $\sim 20 \mu \mathrm{m}$, as observed also in silicon-supported lipid multilayers. ${ }^{39}$ This demonstrates that local photoemission alone is not sufficient to explain the observed charging phenomena. In addition, Compton scattering in the bulk substrate can 


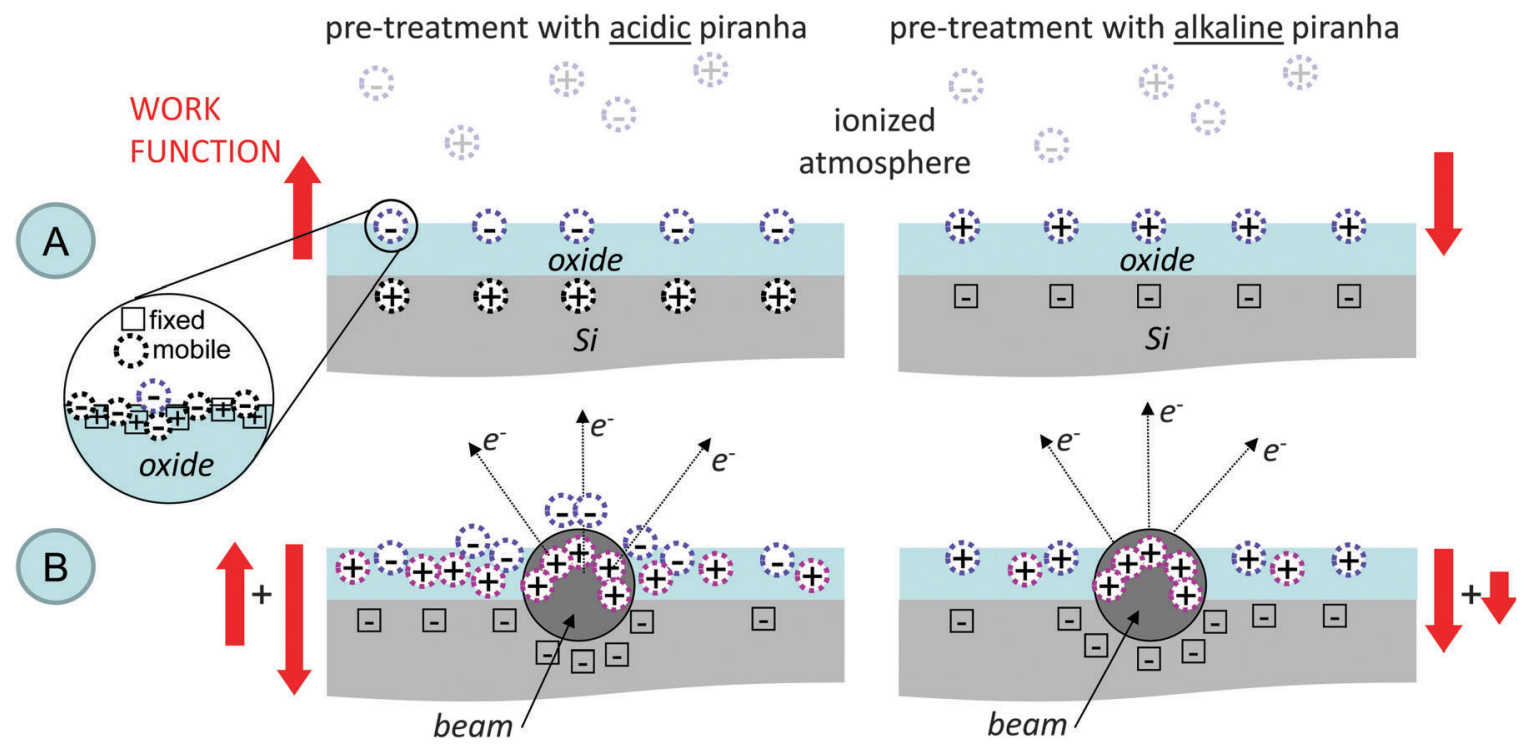

Fig. 3 Schematic representation for p-type Si of the main types of fixed (squares) and mobile (circles) charges present at the surface and in the bulk semiconductor beneath the surface oxide layer (mirror charges). (A) Beam below the sample surface. (B) Beam at the surface. Left column: acidic treatment. Right column: alkaline treatment. The red arrows qualitatively represent the observed sign and magnitude of the work function variations when the beam is first switched on (A) and when it reaches the surface (B).

enlarge the lateral width of the X-ray dose profile delivered by the direct micro-beam, but it cannot be invoked as the main origin for the macroscopically extended $(\sim 1 \mathrm{~mm})$ surface charging detected by the Kelvin probe. Finally, after beam switching off, the work function at the beam position is always slightly lower than anywhere else. This indicates permanent residual positive charging of the surface area that is directly irradiated by the X-ray beam.

While it is not surprising that intense high energy X-ray irradiation can cause permanent positive charging at the beam footprint, it is not immediately apparent why surface charging induced by ionized air molecules is almost reversible/irreversible for acidic/alkaline pre-treatments. We attribute this phenomenon to differences in surface charge mobility. As known from the ion transport mechanism at insulator/metal interfaces,$^{24}$ mobile surface charges move together with mirror charges present in the bulk semiconductor beneath the native oxide layer. While the majority charge carriers of p-type silicon (holes) facilitate the discharging of the negative charges absorbed from air on the acidically treated surface, the dissipation of positive mobile ionized air charges absorbed on the alkaline treated surface requires negative mirror charges, which are lacking in p-type Si. In order to confirm this hypothesis, the same X-ray beam exposure protocol was applied to an acidically treated n-type $\mathrm{Si}$ wafer, where the majority charge carriers (electrons) cannot contribute to the dissipation of the mobile negative surface charges absorbed from air. Indeed Fig. 4 demonstrates that while the charging behavior is similar, the discharging of n-type $\mathrm{Si}$ after beam switching off (Fig. $4 \mathrm{~B}, t \sim 3200 \mathrm{~s}$ ) is much slower than the instantaneous discharging exhibited by p-type Si (Fig. 4A, $t \sim 3400 \mathrm{~s}$ ).

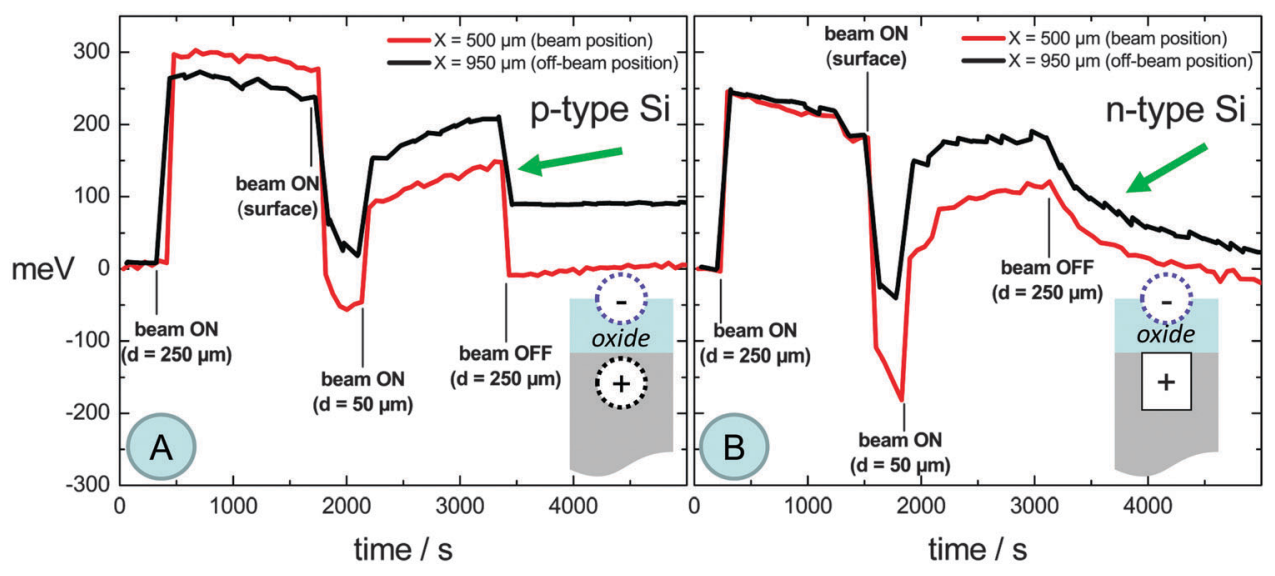

Fig. 4 Comparison of work function evolution at beam (red lines) and off-beam (black lines) positions for acidically treated (A) p-type Si and (B) $n$-type Si. Note the slower decay (green arrows) of the reversible negative charging for n-type Si after beam switching off ( $t>3200 \mathrm{~s}$ ). 


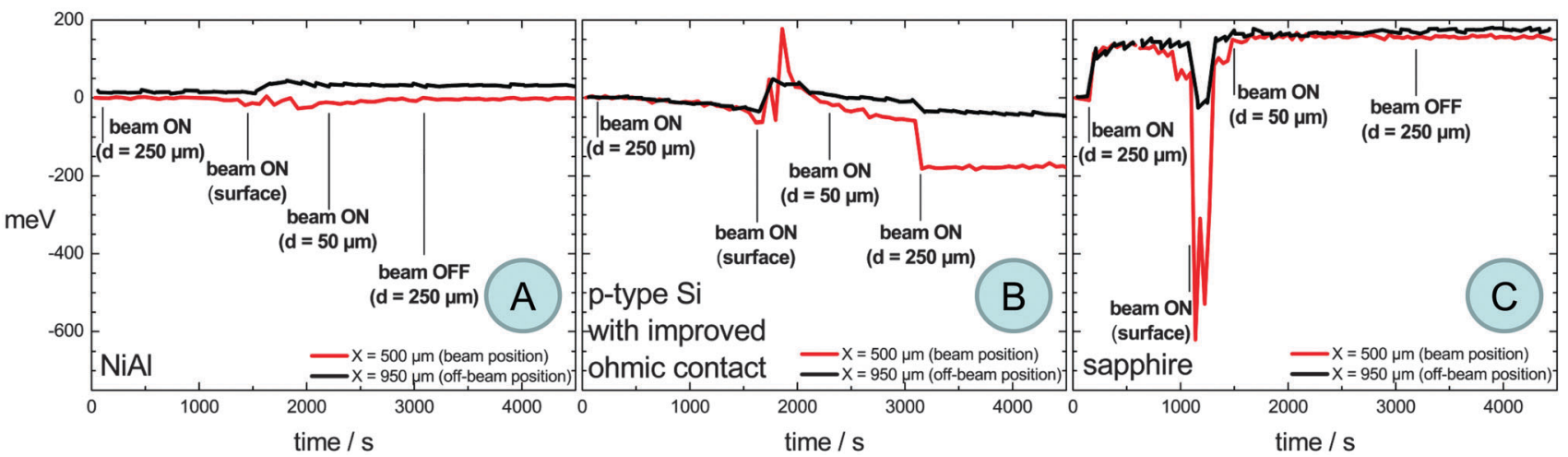

Fig. 5 Effect of varying substrate nature and ohmic contact quality. Comparison of work function evolution for (A) NiAl conductor, (B) p-type Si semiconductor with gold layer bottom coating, and (C) sapphire insulator.
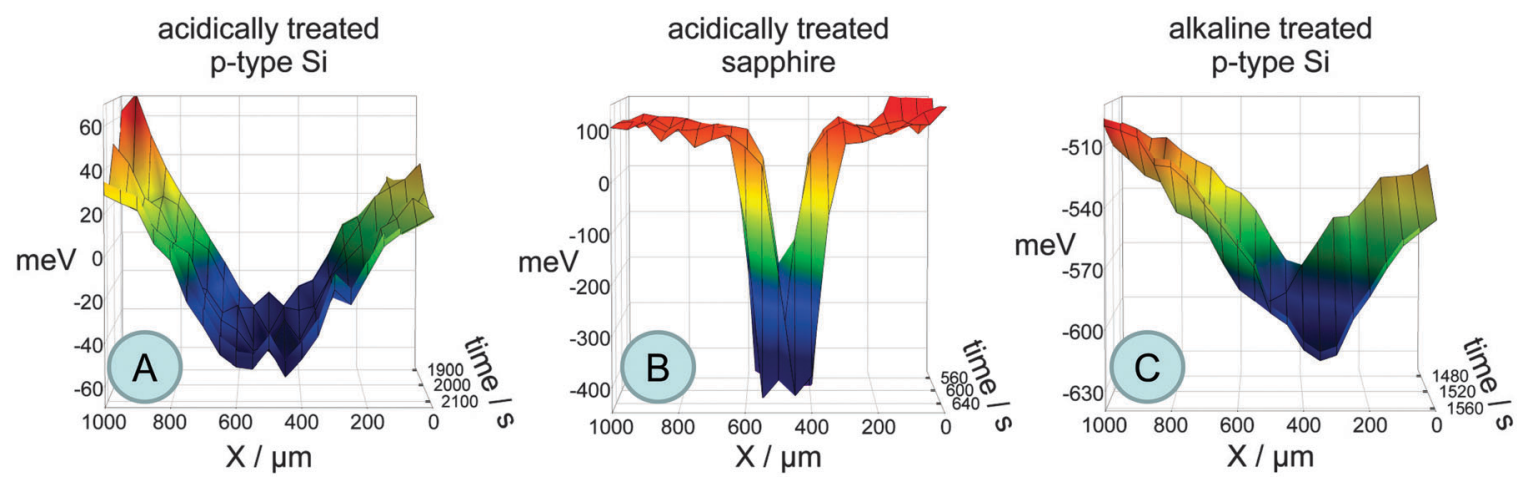

Fig. 6 Evolution of the work function profiles when the X-ray beam impinges the surfaces of the $p$-type Si (A and C) and the sapphire (B). The deeper and more localized work function trench exhibited by sapphire is a consequence of its lower bulk conductivity.

Our investigations of X-ray-induced surface charging phenomena were completed by applying the same experimental protocol to other samples belonging to three different groups in terms of conductivity: a conductor (NiAl alloy), a semiconductor (p-type silicon) with improved grounding ensured by an evaporated gold layer at its bottom, and an insulator (sapphire). The corresponding work function traces at beam (red) and off-beam (black) positions for different beam depths $d$ are presented in Fig. 5. For the NiAl alloy (Fig. 5A), no significant response is detected as charges quickly dissipate or are compensated by corresponding counter-charges. Small effects are however still discernible, thus confirming the high sensitivity of our approach. The gold-coated p-type silicon as well exhibits only minor variations (Fig. 5B), indicating the role of the improved grounding for enhanced charge dissipation. However, after beam switching-off, the photoemission-induced permanent positive charging at the beam footprint is more evident than in the un-grounded case (Fig. 1C), due to the improved dissipation (by grounding) of the mobile negative charges absorbed from air. In the insulator case represented by sapphire (Fig. 5C), the acidic treatment $(\mathrm{pH} \sim 8$ for sapphire IP) induces a positive work function shift at beam switching-on as observed for $\mathrm{Si}$ (Fig. 1B and 4). However, two differences with respect to $\mathrm{Si}$ samples are noticeable when the beam reaches the surface of sapphire: the work function decrease is stronger $(\sim 700 \mathrm{meV})$ at the beam footprint $(x=500 \mu \mathrm{m})$ and weaker $(\sim 200 \mathrm{meV})$ at the off-beam position $(x=950 \mu \mathrm{m})$. Fig. 6B reports the resulting deeper and more localized work function trenches measured for sapphire during direct surface irradiation, as compared with the corresponding data for p-type $\mathrm{Si}$ (Fig. 6A and C). The broader Si profiles are due to the higher mobility of the negative ionized air charges, attracted to the surface by photoemission-induced positive surface charging, and mirrored by mobile holes in the bulk Si substrates. Contrary to the silicon case, at beam switchingoff $(t \sim 3200 \mathrm{~s}$ in Fig. 5C) no changes are observed in the sapphire work function: the negative charging of the surface is completely retained since there are no mobile positive charge carriers in the bulk sapphire insulator.

\section{Conclusions}

Synchrotron beams cause surface charging not only as a consequence of direct irradiation, but also indirectly, through interactions between the surfaces and partially ionized ambient species. The extent of the resulting work function variations depends crucially on the chemistry of the investigated surfaces, the bulk conductivity of the samples, as well as the quality of their electrical grounding. Optimization of all these factors allows controlling surface charging effects, which are monitored in situ 
by integrating SKP apparatuses with synchrotron beamlines. The results presented here are relevant to all surface and interface science investigations employing ionizing radiation, and are particularly pertinent whenever the sample is in contact with standard air or specifically controlled gaseous or liquid phases.

\section{Acknowledgements}

We acknowledge PSCM support and excellent working conditions provided at ID15A of ESRF.

\section{References}

1 S. Ferrer and Y. Petroff, Surf. Sci., 2002, 500, 605-627.

2 G. Ice, J. Budai and J. W. L. Pang, Science, 2011, 334, 1234-1239.

3 P. J. Eng, T. P. Trainor, G. E. Brown, G. A. Waychunas, M. Newville, S. R. Sutton and M. L. Rivers, Science, 2000, 288, 1029-1033.

4 A. I. Frenkel, C. W. Hills and R. G. Nuzzo, J. Phys. Chem. B, 2001, 105, 12689-12703.

5 J. A. Rodriguez, G. Liu, T. Jirsak, J. Hrbek, Z. Chang, J. Dvorak and A. Maiti, J. Am. Chem. Soc., 2002, 124, 5242-5250.

6 C. C. F. Blake and D. C. Phillips, Biological Effects of Ionizing Radiation at the Molecular Level. Symposium. International Atomic Energy Agency, International Publications, Inc, Vienna, 1962, pp. 183-191.

7 K. D. Watenpaugh, Curr. Opin. Struct. Biol., 1991, 1, 1012-1015.

8 A. Gonzalez and C. Nave, Acta Crystallogr., Sect. D: Biol. Crystallogr., 1994, 50, 874-877.

9 C. Nave, Radiat. Phys. Chem., 1995, 45, 483-490.

10 A. Cheng and M. Caffrey, Biophys. J., 1996, 70, 2212-2222.

11 M. Weik, R. Ravelli, G. Kryger, S. McSweeney, M. L. Raves, M. Harel, P. Gros, I. Silman, J. Kroon and J. L. Sussman, Proc. Natl. Acad. Sci. U. S. A., 2000, 97, 623-628.

12 M. Mezger, H. Reichert, S. Schöder, J. Okasinski, H. Schröder, H. Dosch, D. Palms, J. Ralston and V. Honkimäki, Proc. Natl. Acad. Sci. U. S. A., 2006, 103, 18401-18404.

13 J. Yano, J. Kern, K.-D. Irrgang, M. Latimer, U. Bergmann, P. Glatzel, Y. Pushkar, J. Biesiadka, B. Loll, K. Sauer, J. Messinger, A. Zouni and V. Yachandra, Proc. Natl. Acad. Sci. U. S. A., 2005, 102, 12047-12052.

14 S. Suzuki, in Electronic Properties of Carbon Nanotubes, ed. J. M. Marulanda, 2011, pp. 329-352.

15 S. Suzuki and Y. Kobayashi, Chem. Phys. Lett., 2006, 430, 370-374.

16 T. Y. Teng and K. Moffat, J. Synchrotron Radiat., 2000, 7, 313-317.
17 D. Ehre, E. Lavert, M. Lahav and I. Lubomirsky, Science, 2010, 327, 672-675.

18 J. G. Fraaije, J. M. Kleijn, M. van der Graaf and J. C. Dijt, Biophys. J., 1990, 57, 965-975.

19 C. Satriano, M. Manso, G. L. Gambino, F. Rossi and G. Marletta, Biomed. Mater. Eng., 2005, 15, 87-99.

20 M. Mezger, H. Schröder, H. Reichert, S. Schramm, J. S. Okasinski, S. Schöder, V. Honkimäki, M. Deutsch, B. M. Ocko, J. Ralston, M. Rohwerder, M. Stratmann and H. Dosch, Science, 2008, 322, 424-428.

21 B. Salgin, D. Vogel, D. Pontoni, H. Schröder, B. Schönberger, M. Stratmann, H. Reichert and M. Rohwerder, J. Synchrotron Radiat., 2012, 19, 48-53.

22 L. Kelvin, Philos. Mag., 1898, 46, 82-120.

23 M. F. Becker, A. F. Stewart, J. A. Kardach and A. H. Guenther, Appl. Opt., 1987, 26, 805-812.

24 M. Rohwerder, S. Isik-Uppenkamp and M. Stratmann, Electrochim. Acta, 2009, 54, 6058-6062.

25 D. Mao, A. Kahn, G. L. Lay, M. Marsi and Y. Hwu, Appl. Surf. Sci., 1992, 56-58, 142-150.

26 D. Mao, M. Santos, M. Shayegan, A. Kahn and G. L. Lay, Phys. Rev. B: Condens. Matter Mater. Phys., 1992, 45, 1273-1283.

27 D. Mao, A. Kahn, G. L. Lay, M. Marsi and Y. Hwu, J. Vac. Sci. Technol., B: Microelectron. Nanometer Struct.-Process., Meas., Phenom., 1991, 9, 2083-2089.

28 D. Mao, A. Kahn, M. Marsi and G. Margaritondo, Phys. Rev. B: Condens. Matter Mater. Phys., 1990, 42, 3228-3230.

29 P. Chiaradia, J. E. Bonnet, M. Fanfoni, C. Goletti and G. Lampel, Phys. Rev. B: Condens. Matter Mater. Phys., 1993, 47, 13520-13526.

30 H. Ren, H. Sinha, A. Sehgal, M. T. Nichols and G. A. Antonelli, Appl. Phys. Lett., 2010, 97, 072901.

31 J. L. Lauer and J. L. Shohet, IEEE Trans. Plasma Sci., 2005, 33, 248-249.

32 H. Reichert, V. Honkimäki, A. Snigirev, S. Engemann and H. Dosch, Phys. B, 2003, 336, 46-55.

33 F. Venturini, S. Schöder, W. F. Kuhs, V. Honkimäki, L. Melesi, H. Reichert, H. Schober and F. Thomas, J. Synchrotron Radiat., 2011, 18, 251-256.

34 Y. Duval, J. Mielczarski, O. Pokrovsky, E. Mielczarski and J. Ehrhardt, J. Phys. Chem. B, 2002, 106, 2937-2945.

35 A. Shchukarev, J. Rosenqvist and S. Sjoberg, J. Electron Spectrosc. Relat. Phenom., 2004, 137, 171-176.

36 M. Kornfeld, J. Phys. D: Appl. Phys., 1976, 9, 1183-1192.

37 M. Rohwerder and A. Michalik, Electrochim. Acta, 2007, 53, 1300-1314.

38 F. G. Donnan, Chem. Rev., 1924, 1, 73-90.

39 S. K. Ghosh, B. Salgin, D. Pontoni, T. Reusch, P. Keil, D. Vogel, M. Rohwerder, H. Reichert and T. Salditt, Langmuir, 2012, 29, 815-824. 